\title{
AutoTEM 5 - Fully Automated TEM Sample Preparation for Materials Science
}

M Dutka $^{1}$ and A Prokhodtseva ${ }^{1}$

1. Thermo Fisher Scientific, Eindhoven, Netherlands.

Preparation of a thin S/TEM sample using Focused Ion Beam is still considered a challenging task, requiring high level of expertise, long training time and unique skills of the operator [1]. AutoTEM 5 is the revolutionary software application for DualBeam ${ }^{\mathrm{TM}}$ systems that fully automates the entire process of lamella preparation - from chunking to in situ lift-out and thinning. It is designed to be reliably applied for work with the wide range of the most difficult materials.

Automated in-situ lamella lift-out has been already widely used for specific use cases in semiconductor industry [2]. AutoTEM 5 is the first application that brings full automation to the complete process of insitu lift-out for the large variety of material science samples, resulting in the most efficient, high throughput S/TEM sample preparation. So in full automation mode thinned lamella could be prepared as fast as $45 \mathrm{~min}$.

While allowing the completely autonomous operation, AutoTEM 5 possesses unique flexibility. First, level of automation could be setup by the user. In this way, user can be guided through the complete workflow, if necessary, which could be beneficial for example for training purposes and better understanding of the workflow. Then, editable templates allow optimization of milling strategies for materials that are difficult to handle due to hardness, surface structure or other complicating factors. A configuration of any however difficult and unconventional sample could be setup using the template features. Once a template for every sample is selected, the user only needs to choose positions in the bulk sample from where lamellae will be extracted and define the positions for the attachment on the TEM grid or grids. Lamellae will be automatically processed one by one.

Overall, AutoTEM 5 enables even newer users to utilize the top system performance and makes throughput and reproducibility of TEM sample preparation available to both experienced and newer users.

References:

[1] LA Giannuzzi et al., Introduction to Focused Ion Beams (Springer).

[2] R Alvis et al., 38th International Symposium for Testing and Failure Analysis (2012) p. 391. 


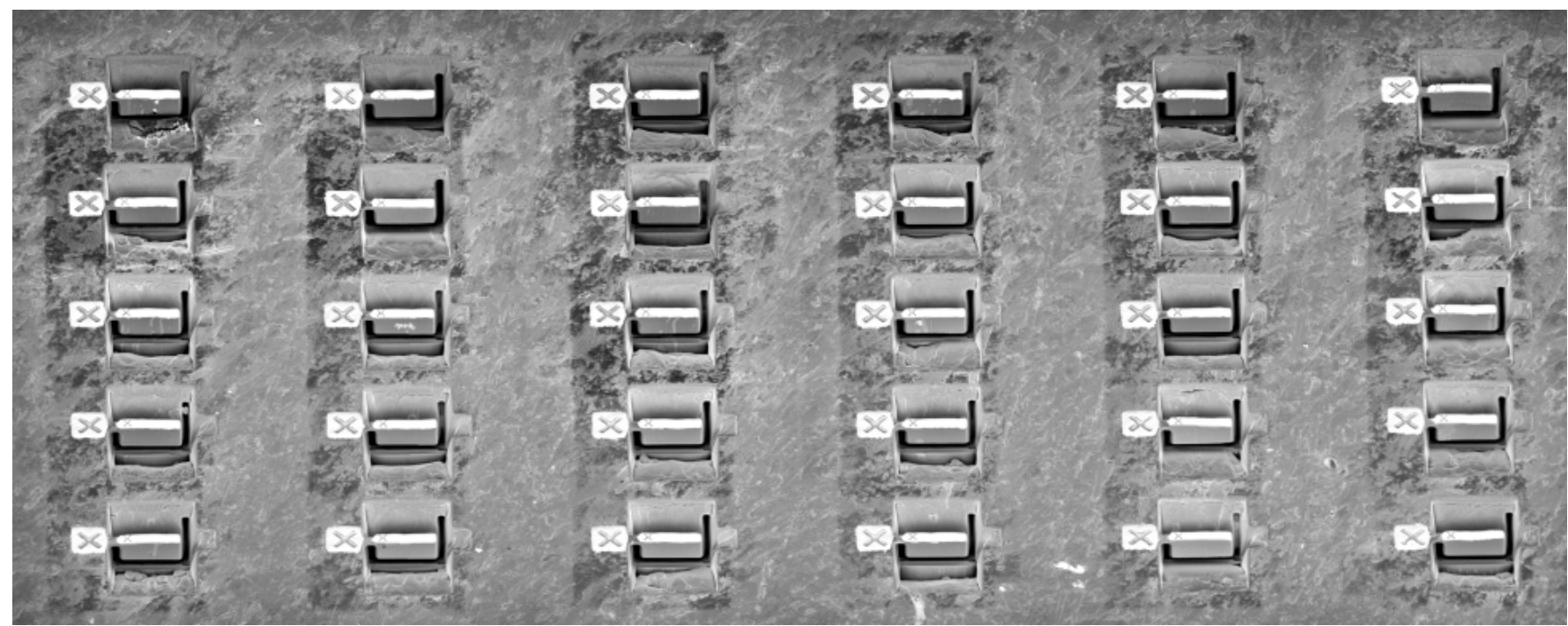

Figure 1. Aluminum sample, where a 5x6 array of S/TEM lamellas has been prepared with AutoTEM software fully unattended, undercut and ready for lift-out in 6 hours. The software allows automatically defining the array and shifting individual locations, to more precisely position the lamella.

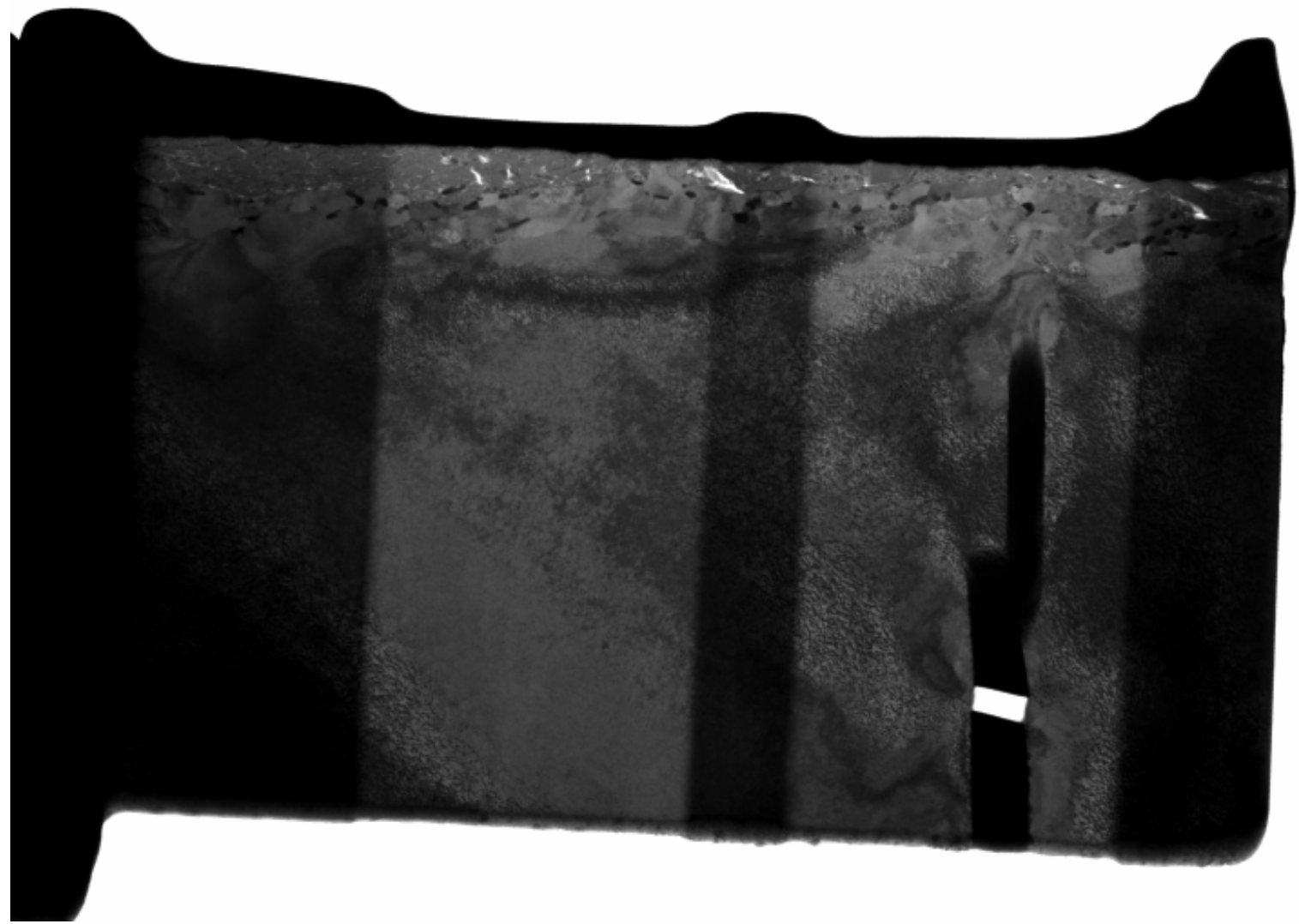

Figure 2. An example of a finished TEM sample with 2 thinned windows. This BF-STEM image acquired on Thermo Scientific DualBeam shows an excellent thickness uniformity over the whole thinned area. 